\title{
A METHOD FOR THE STUDY OF PHOSPHORESCENT SULPHIDES.
}

By Fred. E. Kester.

WiTH such phosphorescent materials as are luminous for only phoroscope for photometric measurements. A large part of his quantitative study of phosphorescence was upon this kind of materials. To obtain measurements on substances, which continue to emit light for a number of minutes, and in some cases for several hours, after illumination, he made direct observations by adjusting the intensity of his comparison surface to equality with the transient intensity of the test substance and noting the instant at which the adjustment was made.

So far as I have been able to find, later observers, notably $\mathrm{L}$. Darwin, ${ }^{2}$ E. Wiedemann, ${ }^{3}$ H. Becquerel ${ }^{4}$ and C. Henry, ${ }^{5}$ have used these two methods with slight modifications to suit special needs.

\section{Apparatus.}

In order to study the luminosity of sulphides of the alkaline earths between the limits imposed, on the one hand, by the intermittent character of the observations when the phosphoroscope is used, and on the other by the difficulty of making, by direct observation, a measurement of intensity of phosphorescence very soon after an illumination, I used the following device. A vertical cylinder of the test substance was rotated about its axis and was illuminated on one side through an aperture which was placed quite close to the cylinder. Then the emitted light was examined by a spectro-

1 La Lumière, I, p. 244.

${ }^{2}$ Chem. News, 42, p. 302, I880 ; Phil. Mag., V-II, p. 210, I88I.

${ }^{3}$ Wied. Annalen, 37, p. I77, I889; Phil. Mag., V-28, p. I49, I889; Eder's Jahrbuch f. Photographie, 5, p. 587.

${ }^{4}$ Comptes Rendus, II3, pp. 618-672, I89r.

5 Comptes Rendus, II 5, pp. 505-602, I892. 
meter, the slit of which was diametrically opposite the illuminating aperture. With a constant intensity of illuminating light and a constant speed of rotation, however slow, the intensity of the emitted light, as observed in the spectrometer, would, of course, remain the same, so that there would be no difficulties in making spectrophotometric comparisons, aside from those which necessarily accompany work with very weak intensities.

It was my first intention to get the test substance into cylindrical form by filling a piece of glass tubing with it; this method would have left the phosphorescent powder in its natural form. But I was unable to find a piece of tubing which did not diffuse into the spectrometer some of the light carried around from the aperture by the internal reflections within the glass. The diffusion was caused by air bubbles and milky streaks inside the glass.

I finally decided to fix upon the convex surface of a turned brass cylinder, $27 \mathrm{~mm}$. in diameter, a thin layer of the sulphide, by mix . ing a small quantity of it with a few drops of varnish and enough turpentine to make the mixture of the proper consistency, and then painting the convex surface with it. Varnish and turpentine were chosen for this purpose because neither of them has any tendency to reduce the sulphide to a sulphate by oxidation and because the varnish, when dry, protects the material from this oxidizing action of the moisture of the air. The fact that the powder, when so treated, was far from its natural state was a serious objection to this method.

The exciting light (from an arc lamp or from the sun) was formed into a spectrum and the cylinder with its aperture was placed in that part where the efficient wave-lengths lay. It was then necessary to measure the energy in the region of the spectrum which was used in exciting phosphorescence. The usual methods of the thermopile and bolometer, all of which necessitate the use of a delicate galvanometer, were not available because of magnetic disturbances; and a photometric method was not convenient. After a number of attempts to devise a suitable method, it was suggested to me that a Crook's radiometer be built in the form which Mr. E. F. Nichols ${ }^{1}$ had used, in some measurements of radiations of long wave-length, 
while in Berlin. This was done, the piece being made very similar in form to his.

It may be of sufficient interest to mention here a difficulty which I encountered in the process of construction of the radiometer. In a tentative form of the apparatus a metal top, with a vertical brass tube soldered into it, was used; into the brass tube was sealed the tube of a glass stop-cock by means of ordinary sealing wax. A number of observers had had trouble with sealing wax when used in the joints of high vacuum pieces, but from all the statements noticed the trouble seemed to arise from leakage. This it was hoped to avoid by making between the glass and brass tubes (the brass being on the outside) a long, firm seal. However, in this form, the enclosure could not be maintained at a sufficiently low pressure, and a search for leaks was commenced. After some time it was noticed that the pressure did not rise steadily, but came up to a certain value and then hung there, indicating the presence of a vapor tension rather than of a leak. The sealing wax was suspected at once and in order to try it a few small pieces were placed in a clean vacuum tube which was then pumped to a high vacuum and sealed off. The pressure in the tube (indicated by the character of electric discharge through it) rose to one or two tenths of a millimeter in about half an hour and then remained constant.

A glass top was then ground to fit the metal body of the radiometer, and the tube of the glass stop-cock welded to it. Even in this final form the piece leaked a little, so that it was necessary to standardize it from day to day by means of a constant source of radiation. A Hefner photometric lamp was used for this purpose.

The arrangement of pieces was, then, as shown in Fig. r. The aperture $A$ in the wall of the dark room was illuminated from without by an arc lamp, with suitable condensing lens, or by sunlight, and the spectrum of the light brought to a focus with the blue end on the cylinder $T$. The mirror $M$ had its upper edge on the same level with the lower edge of the aperture $A$, and was so placed as to reflect light, into the radiometer, of the same wave-length as that used in exciting phosphorescence, the spectrum being so adjusted as to cover both $M$ and $A$.

The standard for photometric comparisons was a magnesium 
oxide surface illuminated by an acetylene flame which was furnished with gas at constant pressure. The light diffused from the surface reached the spectrometer through two Nicol prisms, $P$ and $A$, and

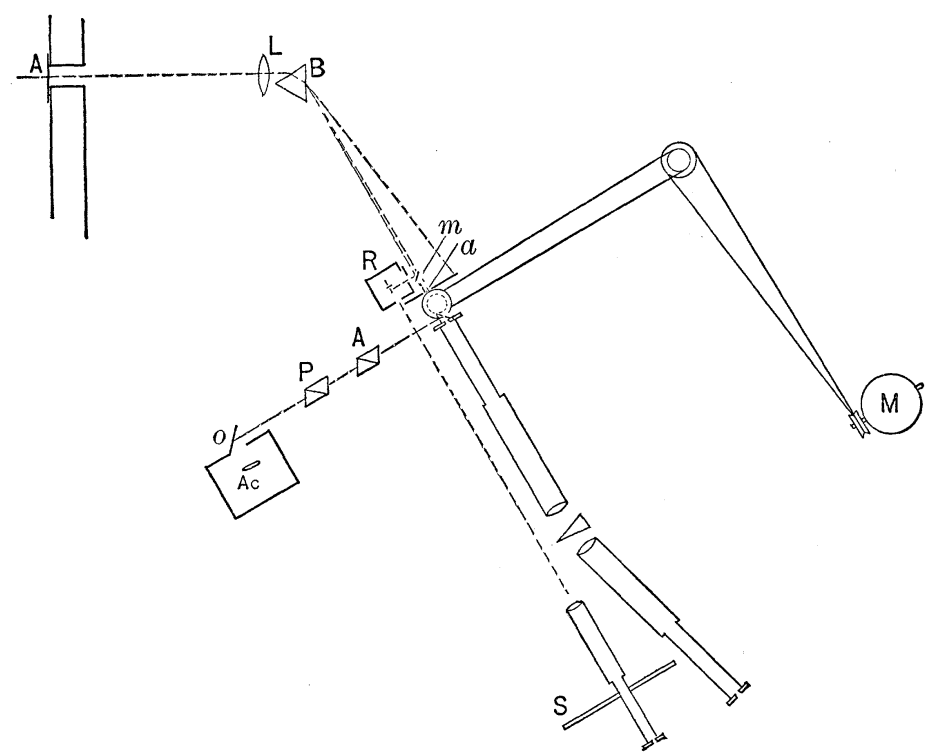

Fig. 1.

a total reflection prism which covered the lower half of the collimator slit. The upper half of this slit received light from the phosphorescent cylinder.

Finally, the spectra of the phosphorescent light and of the diffused light from the magnesium oxide surface were formed, one above the other in the field of the eye-piece of spectrometer, by a low dispersion prism of crown glass with a $30^{\circ}$ angle. It was necessary to use such small dispersion as this prism gave because of the feebleness of phosphorescent light. The eye-piece contained the usual narrow aperture for cutting out of view all parts of the spectra except those which it was desired to compare. Then when the chosen parts of the spectra were made of the same intensity by adjusting one of the Nicol prisms, the intensity of phosphorescence at this wave-length was measured by $\sin ^{2} \theta$, where $\theta$ is the angle between this position of the Nicols and the crossed position. 
The cylinder $T$ was rotated at a constant speed by a small motor $M$ driven by current from storage cells. By the use of a system of pulleys the speed could be varied from about 0.03 sec. to 7 or 8 sec. per revolution.

As soon as readings were commenced it was found that the arc lamp as a source of exciting light would have to be discarded; its fluctuations were much too rapid to be followed by the luminosity of the sulphide. Observations could be taken only with sunlight on clear days.

On account of the late date at which the necessary apparatus was completed and, too, because of the scarcity of clear sunshine, I have been unable to make observations on more than one materialcalcium sulphide.

\section{Observations.}

To afford a means of correcting for uncontrollable variations in the intensity of the exciting light it was necessary to establish, first of all, a relation between the intensity of phosphorescence and the

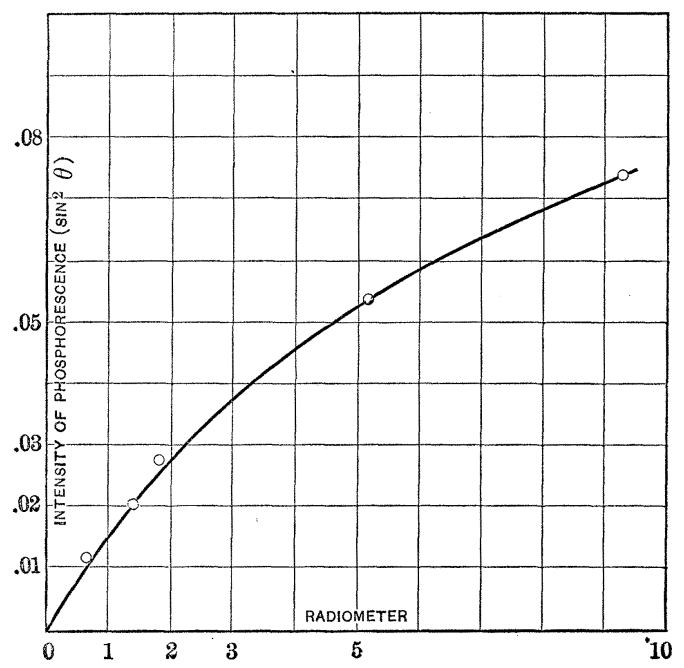

Fig 2.

intensity of illumination. The intensity of the exciting light was varied by interposing pieces of blue glass in the path of the ray of sunlight and was measured by the deflections of the radiometer, Mr. 
Nichols having found that, under proper conditions, the deflections were proportional to the energy of radiations.

In obtaining these preliminary readings, the carbon bisulphide prism, which was used in order to obtain sufficient separation of the

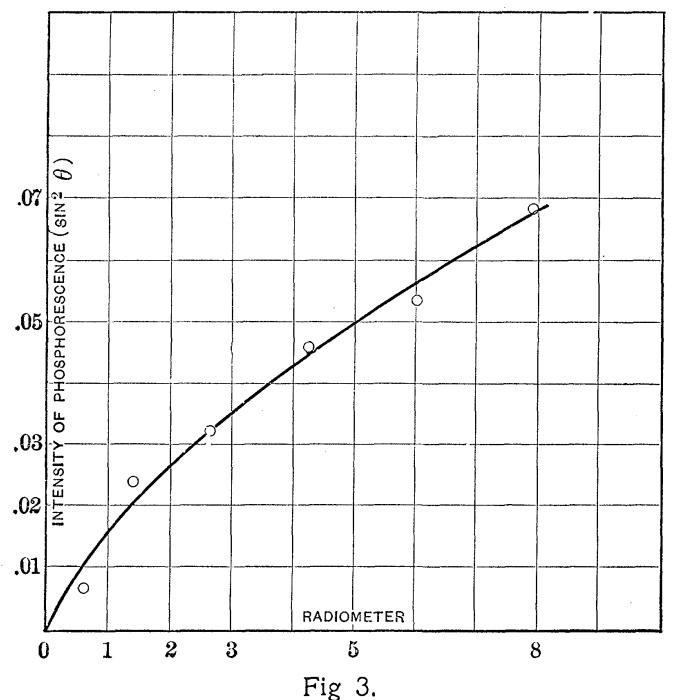

wave-lengths of sunlight within the necessarily short distance $B T$, gave some trouble. The action of the light in traversing the prism decomposed the bisulphide, setting free small particles of sulphur which diffused light in all directions. Some of this stray light, traveling in the direction of $m$ and $a$ caused an increase in the deflection of the radiometer but no corresponding increase in the luminosity of the phosphorescent material. A couple of curves obtained with bad charges of bisulphide are shown in Figs. 2 and 3.

Each point is obtained from averages of from three to five readings of the radiometer and of the Nicol prisms. To obtain the intensity of phosphorescence from the Nicol prism readings it is sufficiently accurate to take the square of the sine of the average angle.

A set of readings taken with a fresh charge of bisulphide is given in the table following; it shows to what degree of accuracy the settings of the Nicols were made. The errors in this column of readings made it desirable to have a larger number of observations in each group, but time did not permit. 
[VoL. IX.

\begin{tabular}{|c|c|c|c|c|c|}
\hline \multicolumn{3}{|c|}{ Radiometer. } & \multicolumn{3}{|c|}{ Nicol Prisms. } \\
\hline $\begin{array}{c}\text { Readings. } \\
11.4 \\
11.3 \\
11.4\end{array}$ & $\begin{array}{c}\text { Zero. } \\
3.9\end{array}$ & $\begin{array}{c}\text { Deflection. } \\
7.47\end{array}$ & $\begin{array}{c}\theta \\
18.0 \\
16.0 \\
16.8\end{array}$ & $\begin{array}{l}\text { Av. } \theta . \\
16.9\end{array}$ & $\begin{array}{c}\operatorname{Sin}^{2} \text { (Av. } \theta_{.} \text {) } \\
.0853\end{array}$ \\
\hline $\begin{array}{l}10.8 \\
10.7 \\
10.7 \\
10.8\end{array}$ & 4.0 & 6.75 & $\begin{array}{l}16.8 \\
15.4 \\
16.2 \\
15.8\end{array}$ & 16.05 & .0765 \\
\hline $\begin{array}{l}9.1 \\
9.2 \\
9.1 \\
\end{array}$ & 4.0 & 5.13 & $\begin{array}{l}13.0 \\
12.8 \\
12.8\end{array}$ & 12.87 & .0500 \\
\hline $\begin{array}{l}7.4 \\
7.2 \\
7.2 \\
\end{array}$ & 4.0 & 3.27 & $\begin{array}{l}11.8 \\
12.2 \\
11.8\end{array}$ & 11.93 & .0432 \\
\hline $\begin{array}{l}6.9 \\
6.9 \\
6.9 \\
\end{array}$ & 4.0 & 2.90 & $\begin{array}{r}10.2 \\
9.8 \\
10.2 \\
\end{array}$ & 10.07 & .0315 \\
\hline $\begin{array}{l}6.2 \\
6.1 \\
6.3 \\
6.2 \\
\end{array}$ & 4.0 & 2.20 & $\begin{array}{l}8.2 \\
9.0 \\
7.8 \\
8.0\end{array}$ & 8.25 & .0210 \\
\hline $\begin{array}{l}5.1 \\
5.1 \\
5.1\end{array}$ & 4.0 & 1.10 & $\begin{array}{l}6.2 \\
7.4 \\
6.8\end{array}$ & 6.80 & .0140 \\
\hline
\end{tabular}

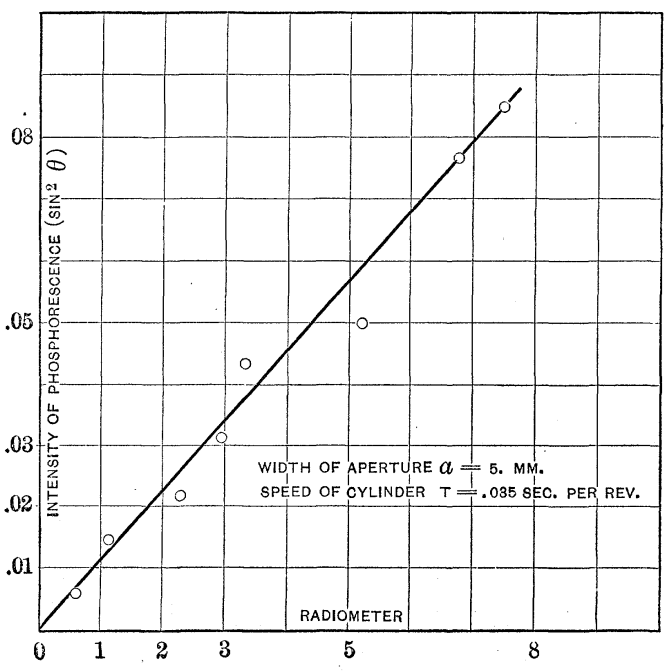

Fig 4.

The curve of Fig. 4 is plotted from this set of readings and indicates that the luminosity of the sulphide is proportional to the 
intensity of illumination, under the conditions of the present apparatus. Fig. 5 gives another curve of the same type, taken with a different width of aperture $a$ and slightly different position of the spectrum of sun light. For all wave-lengths of exciting light, widths

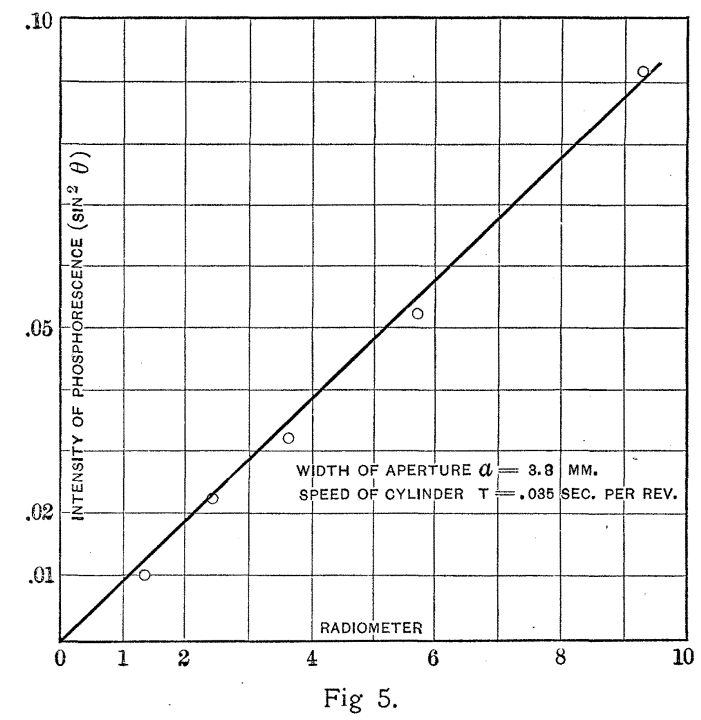

of aperture $a$, and speeds of rotation which were tried it was found that the curves of intensities were straight lines.

Then keeping the intensity of illumination constant, or correcting, by means of the above curves, for the uncontrolable variations, and keeping the speed of rotation constant, the effect of varying the width of $a$ was determined. During these observations the intensity of the exciting light would, of course, vary somewhat. The variation was corrected for by dividing $\sin ^{2} \theta$ by the radiometer deflection, which operation is directed by the proportionality existing between the intensities. Fig. 6 is a curve showing the relation between the luminosity and width of aperture.

The relation between intensity of phosphorescence and speed of rotation of the cylinder is shown by Fig. 7. Here each point is obtained from an average of from eight to ten observations.

With the width of aperture $A$, which it was necessary to use, the spectrum of the sunlight was so impure that most of the wave- 
lengths, which were efficient in producing phosphorescence, were to be found in the aperture $a$. On account of this it was found impossible to obtain accurately by measurements the relation between the luminosity and the wave-length of exciting light. For this purpose a flat surface was painted with the sulphide of calcium and ex-

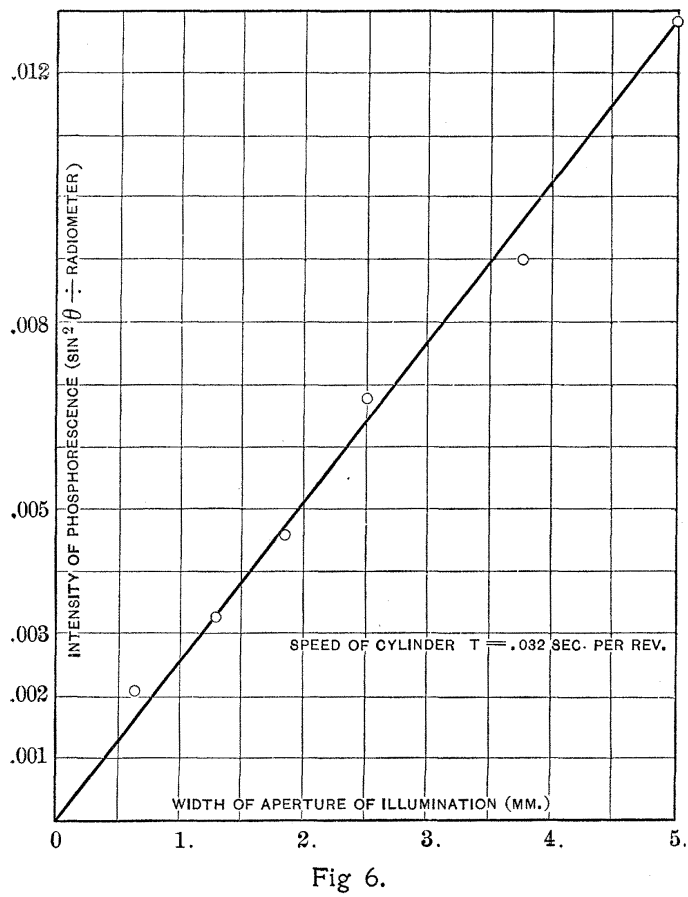

posed in the spectrum from a narrow aperture. By noticing the luminosity soon after illumination a visibility curve was sketched showing approximately the desired relation.

And in the case of constant conditions of the cylinder $T$-as to illumination and speed-it was useless to compare the intensities at various regions of the spectra of the emitted light and of the light from the surface $o$ because of the uneven distribution of energy in the spectrum of the acetylene flame. A visibility curve of intensity and wave-length of phosphorescent light was made.

These last two curves are drawn in the same figure (Fig. 8) in order to show more clearly the relation between the wave-lengths 
of exciting and emitted light. The ordinates of the lower curve represent the intensity of the whole group of wave-lengths of the
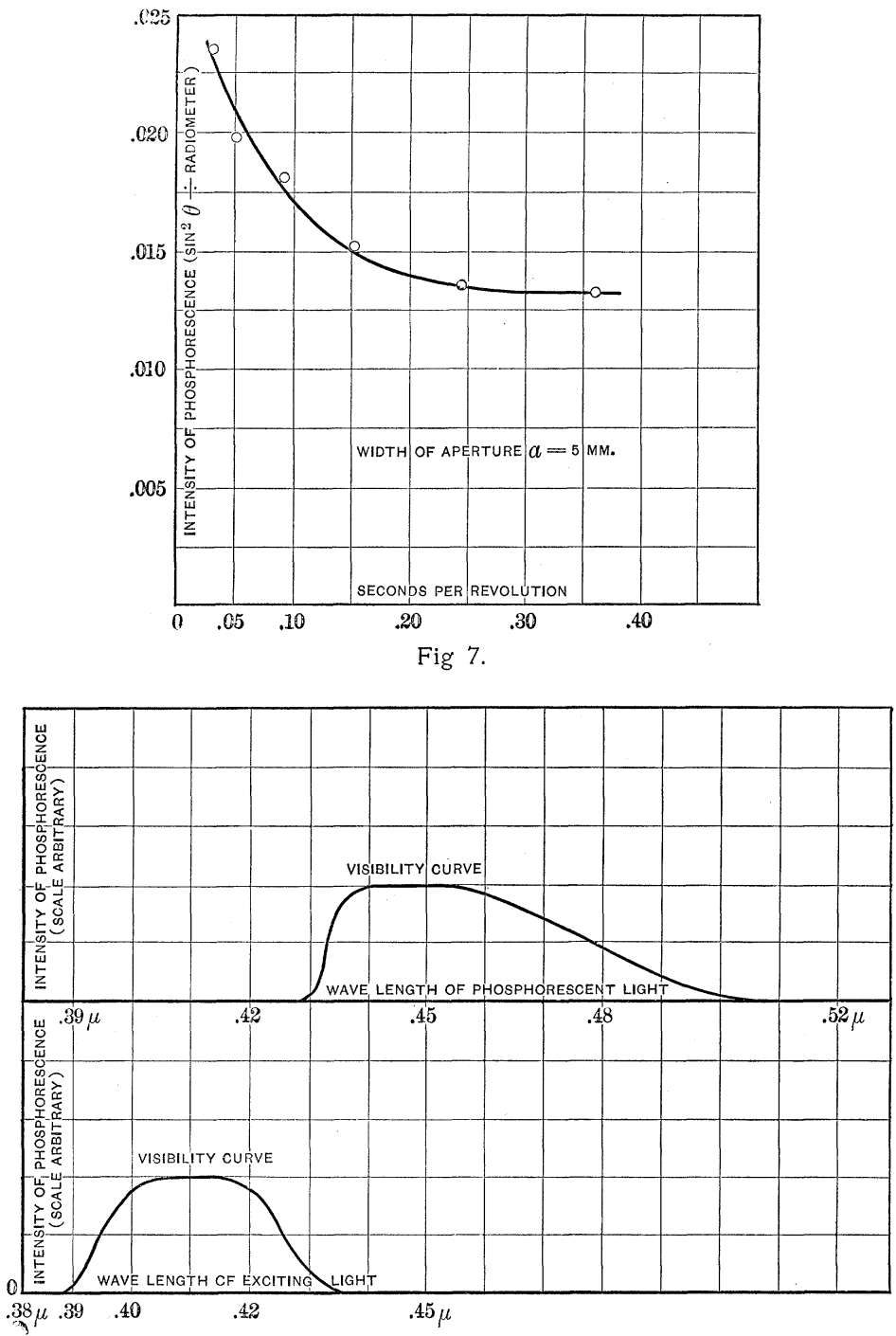

Fig. 8.

phosphorescent light. With a wider dispersion of the illuminating light, giving greater purity of spectrum than the present arrange- 
ment of apparatus affords, and thereby permitting of measurements on the wave-lengths of exciting light, it will be possible to separate this curve into its component curves and, by so doing, show quite definitely the relation between the wave-lengths of exciting and of emitted light. This relation would be of special interest in those sulphides which have more than one band in their spectra.

It may be shown by a discussion of the curve of intensity of phosphorescence and time of revolution of the cylinder, in connection with the curves of phosphorescence and width of aperture, that there are no definite curves of increase and decrease of luminosity during and after illumination; in other words, the rate of increase or of decrease at any intensity of phosphorescence depends upon the previous treatment which the luminous material has undergone. This is a fact which E. Wiedemann ${ }^{1}$ had noticed in the case of Balmain's luminous paint and which he stated to be true of the sulphides of the alkaline earths, either from a knowledge of the constituents of the paint or from independent observations made on these sulphides.

The peculiarity may be shown by means of the present curves as follows: Assume that there are definite curves such as shown in

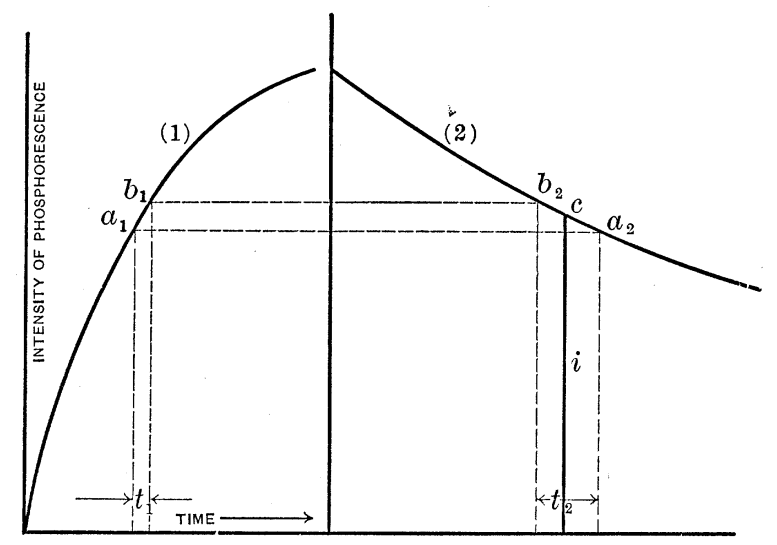

Fig. 9.

Fig. 9, curve (I) being for increase and (2) for decrease of luminosity. Then when the cylinder of material has reached a steady

${ }^{1}$ Weid. Annalen, 37, 177 ; 1889; Phil. Mag., 15-28, 149, 1889. 
condition for a certain speed of rotation and a certain intensity of illumination, the material is going through some such cycle as $a_{1} b_{1}$ $b_{2} a_{2}$ for each revolution of the cylinder. Observation by the spectrometer is made as the material passes the slit, namely, at the condition represented on the curve (2) by the point $c$. Now since the increase in intensity from $a_{1}$ to $b_{1}$ is equal to the decrease from $b_{2}$ to $a_{2}$, the ratio of the slopes of the two curves at this intensity is equal to the inverse ratio of the times of illumination and insulation. But we can work at the same intensity of phosphorescence by decreasing the width of the aperture $a$ and increasing the speed of rotation; in so doing we have changed the above ratio of times and consequently have no determined slope for the curves at the intensity considered.

So far, therefore, as the present observations are concerned the behavior of the calcium sulphide will have to be defined in terms of the apparatus used. There seems to be no way of so combining the results as to show the behavior in a more absolute manner. For this reason it is unnecessary to give the various times of illumination and of insulation in more detail than is done in the preceding pages and accompanying figures.

Dept. of Physics, Cornell Univ., June, 1899. 